\title{
FoodSimplex in restaurants-how can it provide safer meals?
}

\author{
Baltazar $\mathrm{A}^{1,2^{*}}$, Figueiredo $\mathrm{JP}^{3}$, Ferreira $\mathrm{A}^{4}$, Manyes $\mathrm{L}^{5}$ and Mañes $\mathrm{J}^{5}$ \\ ${ }^{1}$ Faculty of Pharmacy, University of Valencia, Spain \\ ${ }^{2}$ Instituto Politécnico de Coimbra, Coimbra Health School-ESTESC, Dietetics and Nutrition Department, Coimbra, Portugal \\ ${ }^{3}$ Instituto Politécnico de Coimbra, Coimbra Health School—ESTESC, Coimbra, Portugal \\ ${ }^{4}$ Instituto Politécnico de Coimbra, Coimbra Health School-ESTESC, Coimbra, Portugal \\ ${ }^{5}$ Department of Public Health. Faculty of Pharmacy. University of Valencia. Spain
}

\begin{abstract}
The need and obligation to provide safe food to consumers leads to implement effective food safety systems along the entire chain production, shipping and distribution, as hazard analysis and critical control points (HAPPC) principles. In Portugal, small and medium restaurants are family business that may lack the knowledge and the money for technical support to follow these principles. The aim of this study is to assess a public health tool, FoodSimplex, in order to compare food safety, good manufacturing practices (GMP) and HAPPC before, during and after its implementation in small and medium restaurants. Results show that, after systematic training and frequent audits, FoodSimplex was linked to a change of habits: an increase of food safety and improvement of hygiene and GMP in small and medium restaurants.
\end{abstract}

\section{Introduction}

The offer increase in mass caterer establishments such as restaurants, canteens, schools, hospitals and catering enterprises [1], arises the concerns, are the meals prepared and ready for consumption by the final consumer safe? Do the food business operators (FBO) have the means and the technical support to respect the European Union regulation? Is there a preventive action plan effective to provide food safety in medium and small companies?

Billions of meals are prepared safely each day throughout the world but $22 \%$ outbreaks of food borne diseases were reported in Europe that had their origin in processed foods and/or by catering establishments $[2,3]$.

It is estimated that millions of people have had a foodborne disease at least once. Health agencies associate these numbers with the consumption of meals in restaurants [4]. The outbreaks of this cases, the results are emotional, physical and financially devastating to all the intervenient, clients and the business management [2].

The mass caterer sector has grown in recent decades and several factors have been identified such as increased number of individuals living in urban areas, distance from home/work, increased percentage of women in the workplace, increased financial power and dietary concerns $[4,5]$.

The economic activity of catering in Portugal embraces (I) the preparation and sale activities of food products for consumption, usually on site or in other establishments that do not produce those products; (II) the activities of preparation of meals or dishes delivered and/or served at the place determined by the customer for a specific event; (III) the supply activities and, where appropriate, the preparation of meals and drinks to well-defined groups of people, like public collectivities (hospitals, schools, elderly places, etc.). It includes canteens and military spaces; it also includes the provision of meals based on a contract for a given time period; (IV) the sales activities of drinks and small meals for consumption on the premises with or without spectacle. (Bank of Portugal, 2011) [6].

The European food laws introduced a new concept in the food market, "from farm to fork", by designing a cross accountability to all stakeholders in the food chain $[3,7]$. The catering sector assigns a very important role to entrepreneurs, considering them primarily responsible for food safety [8].

The European Union (EU) has created legal tools to ensure food hygiene in the sector, as well as official entities in charge of controlling and inspecting establishments to ensure public health [7]. Council Regulations (EC) 852/2004 on hygiene of foodstuffs were created to ensure restaurants must obey with general hygiene requirements [9].

The need and obligation to produce safer food go to the inevitable implementation of effective food safety systems along the entire chain of production, shipping and distribution, namely a system based on the Hazard Analysis and Critical Control Points (HACCP) principles [10].

This preventive system requires a strategic approach to the stages of production /serving, based on the identification of inherent hazards such as biological, chemical and physical hazards [11].

Correspondence to: Baltazar A, Faculty of Pharmacy, University of Valencia, Spain, Instituto Politécnico de Coimbra, Coimbra Health SchoolESTESC, Dietetics and Nutrition Department, Coimbra, Portugal, E-mail: analuciabaltaz-ar@hotmail.com

Key words: food safety, FoodSimplex, restaurants, hygiene, good manufacturing practices

Received: September 01, 2017; Accepted: September 25, 2017; Published: September 29, 2017 
The HACCP system is a preventive system resulting from the application of scientific and technical principles. It is an essential tool for identification and analysis of critical points (CP) at different stages of the process while allowing the establishment of the necessary means to control these points and apply preventive monitoring. The HACCP system stands for proactivity instead of reactivity (corrective approach) [12]. Although manufacturers have used HACCP system successfully for many years it has been less common in small and medium sized enterprises (SMEs), especially those in food service sector. There are considered to be a number of barriers which small businesses find particularly difficult to overcome $[13,14]$.

In Portugal, we have two kinds of situations that can increase the food safety concerns, namely, most of the small and medium restaurants management are from family business, which means that old bad practices and the establishment premises keep going throughout the years. On the other hand, nowadays, like in other European countries, to reduce bureaucracy and facilitate the establishments of the new food business, the restaurant's premises only need registration, without inspection pre-approval, to start working [15]. As so, the restaurants' FBO are responsible for managing hygienic-sanitary quality and for providing safe food to clients and most of the times they don't have the knowledge and the technical support to implement a food safety system or the financial ability to pay external technical care [4].

As so foodborne diseases still continue to be a major public health concern in developed countries due to the number of people affected annually by and the economic losses in this company [3].

This study aims to assess food safety action plan created for medium and small restaurants, named FoodSimplex, as public health tool to be applied by food safety technicians. Is compares food hygiene, good manufacturing practices (GMP) and HACCP documentation before, during and after implementation of FoodSimplex.

\section{Material and methods}

The small and medium restaurants have difficulties in complying the food safety regulations, mainly because of the lack of well-trained personnel, lack of motivation or adhesion to HACCP system on the part of the workers, and the lack of financial and economic resources to address the deficiencies in the facilities.

The small and medium restaurants were selected according to the following criteria:

- Portuguese economic activity code in Portugal for restaurant (financial Portuguese code)

- Turnover (up to 10 million euros)

- Geographic area (Leiria district)

- Restaurants interested in participating in the study

The project was a longitudinal study which took place between March 2010 and December 2014. Out of 42 restaurants eligible, 22 remained in the study for the four-year period.

The data collection instrument for the inspection was an audit checklist, created by food safety technicians after a pre-test applied to 31 restaurants, A checklist template was designed to collect data through visual inspection regarding food handlers and facilities hygiene and GMP as well as through interviewing the person responsible with regard to HACCP aspects (manual of procedures, records, etc.) and it's organized in three modules divided into specific topics (items).
After the pre-test, the food safety technicians reviewed some evaluation topics and insert others and the final checklist was the one applied in this study.

The final checklist consisted of 70 observations, each of which could be answered as "Compliance", "No Compliance" and "Not applicable". Every "Compliance" answer was allocated one point; every "No Compliance" was allocated 0 . The final score for each premises was calculated by summing the points. The maximum score premises could achieve was 70 , the minimum was 0 . The audit required approximately $1.5 \mathrm{~h}$ to complete, depending on the size of the premises. The outcome of the audit was a numeric score. The higher the score, the better premises complied with the requirements of the audit. For this study we only analyze the hygiene and GMP requirements.

The 352 audits were studied according to with the results and their evolution during the application of a FoodSimplex methodology (Table 1).

The FoodSimplex methodology, created for this study, includes four stages (Table 1).

The diagnosis audit focuses on gathering information about the food business to identify any areas of potential improvement in the business's premises and to design the HACCP plan. The $2^{\text {nd }}$ stage was meant to present the diagnosis audit report and the HACCP plan through training activities to the food handlers and the FBO. The training session intends to present the nonconformities, identify the areas of the business that have deficiencies, to appropriate action to correct any lacks and to reinforce food safety knowledge.

The $3^{\text {rd }}$ step involved a systematic and independent assessment to determine whether food safety activities and related results comply with planned arrangements and whether these arrangements are implemented effectively and are suitable to achieve objectives [16]. The audits were supplemented with microbiological analyses towards hygiene and food quality standards. The audit reports are only useful if the food handlers and the FBO review the results, understands the risks addressed and makes risk-reduction decisions based on the results [2]. The $4^{\text {th }}$ stage intended to present the reports in a training session and applicate a food safety improvement plan of the restaurant.

The time schedule of the stages was: a month between the $1^{\text {st }}$ and the $2^{\text {nd }}$ stage and in between $2^{\text {nd }} 3^{\text {rd }}$ and the 4 th stage is three months period. After the 4th stage the methodologic process continues repeating the order starting in the $3^{\text {rd }}$ stage.

The audit results were structure and consider by 3 main groups, namely hygiene, GMP and HACCP documentation, however for this study we are only analyzing the 2 main groups Hygiene and GMP, to understand how effective the FoodSymplex is at the time, because the HACCP documentation was absent so the improvement is total $(100 \%)$. As so only 52 checklist observations were used in this work.

\begin{tabular}{|c|c|}
\hline Stage & Action Plan \\
\hline 1 & Diagnosis audit (checklist) and HACCP data collection \\
\hline 2 & $\begin{array}{l}\text { Diagnosis report } \\
\text { HACCP Plan } \\
\text { Training Action "Hygiene \& Food Safety - Restaurants" }\end{array}$ \\
\hline 3 & $\begin{array}{l}\text { Audit } \\
\text { Microbiological analyses }\end{array}$ \\
\hline \multirow{3}{*}{4} & Audit report \\
\hline & $\begin{array}{l}\text { Training Action "Treatment of non-compliance - audit and microbiological } \\
\text { report" }\end{array}$ \\
\hline & Improvement Plan Application \\
\hline
\end{tabular}


The results of the study were subject to statistical analysis, namely, $\mathrm{McNemar}$ test. For the statistical Inference, we took into account a $95 \%$ confidence level for a random error of up to $5 \%$. The specialized software for treatment of analytical data was the IBM SPSS Statistics software, version 24 .

The results were analyzed by score percentage (0-100) represented by the 2 selected groups. Since it was a continuous study, the data analyzed was based only on comparing the average scores provided between the diagnostic audit $\left(1^{\text {st }}\right)$ and the $4^{\text {th }}$ audit, to have a summary knowledge of the change and improvement of the food safety status during the study.

\section{Results and discussion}

There were 42 restaurants eligible for the research, however only 22 maintained for the 4 years period of this study. The main reason for the decline of the companies was the economic crises in Portugal [17], which a big part of the SMEs didn't survive between 2012 and 2014 [18].

The results were analyzed taking into account the $1^{\text {st }}$ and $4^{\text {th }}$ audit, to assess the evolution in hygiene and GMP practices in this SME restaurants. The results were statistically treated by SPSS and the McNemar test was applied to a confidence of $95 \%$.

\section{Hygiene assessment}

The results of the 24 items evaluated for hygiene were structured according to the checklist (Table 2). In general, all the results between the two evaluation moments showed a maintenance or an improvement in the compliance with hygiene requirements. Compliance was maintained between the 1st and 4th evaluation on the following items: state of sanitation in the serving; personal hygiene (uniforms, gloves, adornments, state of health); general hygiene (obsolete material, non-food); disinfection (washing machine). On the remaining items, there was an improvement in the results for compliance. Positive results for hygiene compliance may be related to customer satisfaction, as they attach an important role to the hygiene of catering establishments. The Sarter and Sarter study [19] also reinforces this aspect, noting that the lack of hygiene leads to economic sanctions, being the loss of customers is the most important one. In the research done by Garayoa, et al. [3], 75\% of the catering establishments were in conformity with the organization and cleaning, as in this study, in the 4th evaluation, all companies were in terms of overall hygiene value between $72.7 \%$ and $100 \%$. Utensils and containers are identified as not cleaned in Portuguese studies [20,21], but was not verified in this work. Also unlike the study by Garayoa, et al. [3], in which only $10 \%$ of the handlers presented the correct uniforms and absence of adornments, in this work $72.7 \%$ fulfilled the personal hygiene requirements and maintained them along the study.

There was a statistically significant change in the state of hygiene in the cooking stage between the first and fourth audits, with $63.6 \%$ non-conformities detected initially and after FoodSimplex application, a $27.3 \%$ reduction in non-conformities $(\mathrm{p}=0.021)$. We can also mention that from the eight companies that have complied, there was an increase of 16 restaurants for compliance (72.8\%). Also in this scope, of the 14 non-compliant companies for the hygiene in cooking, nine restaurants improved their condition. In "General hygiene for the locker rooms there was also a statistically significant change. It was verified that in an initial phase 12 companies (54.5\%) presented non-conformities and of these, $83.3 \%$ passed to conform $(n=10)$. Of the restaurants that in the 1 st evaluation phase were in agreement, none regressed. At the end of the 4 th audit it was found that $9.1 \%$ of the establishments maintained non-conformity.

Also, in the large group of "General hygiene" for the first aid box, it was found that in the first evaluation $59.1 \%$ failed the compliance and that after applying the methodology of this study, $76.9 \%(n=10)$ comply. That means, of the $40.9 \%$ of restaurants that initially had compliance, at the end of the fourth phase $77.3 \%$ were satisfied and $22.2 \%$ maintained non-conformity. These results were also statistically significant $(\mathrm{p}=0.039)$

\section{GMP assessment}

The results evaluated were based on the 28 assumptions selected for the evaluation of the GMP. Compliance was maintained between the evaluations on the following items: reception of the raw material (95.5\%); handling in preparation $(90.9 \%)$ and cooking stages (100\%); treatment of leftovers (100\%) and food sampling (100\%) and in serving dishes and the conformity of food products served, both maintaining $100 \%$.

Veiros, et al. [7] named that the equipment and materials are suitable for the areas or tasks, but they were not clearly identified for the area by colors or sanitized following the contact with raw foods during the work shift. In the present study, this as was verified in the preparation stage, and the restaurants that didn't comply $(n=2)$ maintained along the study.

The preservation of the noncompliance in these items was not a concern for the researchers since the values for compliance are in the order of 90 to $100 \%$.

In this evaluation group, there was a decline in compliance, namely: in the storage of tubers, in the 1st stage with $100 \%$ and in the 4 th phase with $95.5 \%$ and in cold storage for nonconforming products of $86.4 \%$ (1st stage) for $81.8 \%$ (4th phase).

As for the weakening of the room temperature storage of tubers, the researchers took into account the fact that the orders of raw material are made in high quantities, there is no stock rotation for their storage time to be reduced. A further justification for the retrogression is the facilities conditions, that don't have the settings for storage of these products and are often in closets close to non-food products (detergents, napkins, etc.). For storage under cold conditions the increase in the number of nonconformities is due to poor packaging of the products in the cold (ice burns), the break in the cold chain (ice crystals in packages) and the high number of products in the cold equipment, reducing the capacity of temperature distribution in the equipment.

The study by Garayoa, et al. [3] also detected as major deviations in the compliance of the storage stage (70\% in refrigeration, $35 \%$ in freezing and $40 \%$ at room temperature), justifying insufficient space areas, inadequate lighting, and unprotected shelves. Mostly nonconformities for the storage at the room temperature of the nonperishable products were related to the direct contact of the food products with the floor or by the nonphysical separation with cleaning products. All these factors coincide with those detected in this study for the regression of conformities for these points.

There were also statistically significant changes, notably in receiving of products for control $(\mathrm{p}=0.021)$. In the first audit $54.5 \%$ $(\mathrm{n}=12)$ of the companies did not comply and after intervention only $18.2 \%(n=4)$ maintained non-conformity. As eight restaurants passed compliance throughout the study. In the $1^{\text {st }}$ audit the nonconformities detected were related to no verification of quantity, temperature, the 
Table 2. Data for hygiene and GMP in restaurants according with nonconformities (NC) and conformities (C) in audit

\begin{tabular}{|c|c|c|c|c|c|}
\hline \multirow{3}{*}{ Audit Tool Premises - Hygiene } & \multicolumn{2}{|c|}{ 1st Audit } & \multicolumn{2}{|c|}{ 4th Audit } & \multirow{3}{*}{ p } \\
\hline & NC & $\mathbf{C}$ & NC & $\mathbf{C}$ & \\
\hline & $\%(n)$ & $\%(n)$ & $\%(n)$ & $\%(n)$ & \\
\hline Reception-Hygiene of the facilities and equipment & $4,5(1)$ & $95,5(21)$ &, $0(0)$ & $100,0(22)$ & - \\
\hline Room Temperature Storage-Hygiene of the facilities and equipment & $40,9(9)$ & $59,1(13)$ & $18,2(4)$ & $81,8(18)$ & 0,125 \\
\hline Cold Storage- Hygiene of the facilities and equipment & $77,3(17)$ & $22,7(5)$ & $54,5(12)$ & $45,5(10)$ & 0.180 \\
\hline Preparation-Hygiene of the facilities, equipment and utensils & $40,9(9)$ & $59,1(13)$ & $18,2(4)$ & $81,8(18)$ & 0.125 \\
\hline Cooking-Hygiene of the facilities, equipment and utensils & $63,6(14)$ & $36,4(8)$ & $27,3(6)$ & $72,7(16)$ & 0.021 \\
\hline Serving-Hygiene of the facilities, equipment and utensils & $9,1(2)$ & $90,9(20)$ & $9,1(2)$ & $90,9(20)$ & 0.500 \\
\hline Personal Hygiene-Uniforms & $27,3(6)$ & $72,7(16)$ & $27,3(6)$ & $72,7(16)$ & 0.500 \\
\hline Personal Hygiene - Gloves &, $0(0)$ & $100,0(22)$ &, $0(0)$ & $100,0(22)$ & -- \\
\hline Personal Hygiene - Evidence of adornments and/or lack of personal hygiene & $9,1(2)$ & $90,9(20)$ & $9,1(2)$ & $90,9(20)$ & 0.500 \\
\hline Personal Hygiene - Visible diseases &, $0(0)$ & $100,0(22)$ &, $0(0)$ & $100,0(22)$ & -- \\
\hline General Sanitation-Dressing rooms & $54,5(12)$ & $45,5(10)$ & $9,1(2)$ & $90,9(20)$ & 0.002 \\
\hline General Sanitation-Soap and disinfectant dispensers and towel rails & $36,4(8)$ & $63,6(14)$ & $27,3(6)$ & $72,7(16)$ & 0.727 \\
\hline General Sanitation-No manual washbasin with hot and cold water & $68,2(15)$ & $31,8(7)$ & $45,5(10)$ & $54,5(12)$ & 0.227 \\
\hline General Sanitation-First aid kit & $59,1(13)$ & $40,9(9)$ & $22,7(5)$ & $77,3(17)$ & 0.039 \\
\hline General Sanitation - Cloths & $31,8(7)$ & $68,2(15)$ & $27,3(6)$ & $72,7(16)$ & 0.500 \\
\hline General Sanitation - Obsolete material and equipment & $27,3(6)$ & $72,7(16)$ & $27,3(6)$ & $72,7(16)$ & 0.500 \\
\hline General Sanitation - Use/conditions of non-food products & $4,5(1)$ & $95,5(21)$ & $4,5(1)$ & $95,5(21)$ & 0.500 \\
\hline General Sanitation - Sanitary plan compliance & $9,1(2)$ & $90,9(20)$ & $4,5(1)$ & $95,5(21)$ & 0.500 \\
\hline Cleaning \& Disinfection-Dishwashing conditions & $72,7(16)$ & $27,3(6)$ & $40,9(9)$ & $59,1(13)$ & 0.065 \\
\hline Cleaning \& Disinfection-Operational dishwasher equipment &, $0(0)$ & $100,0(22)$ &, $0(0)$ & $100,0(22)$ & -- \\
\hline Cleaning \& Disinfection-Waste containers & $68,2(15)$ & $31,8(7)$ & $36,4(8)$ & $63,6(14)$ & 0.065 \\
\hline Cleaning \& Disinfection - Pest Control & $31,8(7)$ & $68,2(15)$ & $27,3(6)$ & $72,7(16)$ & 0.500 \\
\hline Cleaning \& Disinfection- Waste Treatment & $13,6(3)$ & $86,4(19)$ & $9,1(2)$ & $90,9(20)$ & 0.500 \\
\hline Cleaning \& Disinfection - Chemical Products Storage & $36,4(8)$ & $63,6(14)$ & $22,7(5)$ & $77,3(17)$ & 0.500 \\
\hline \multicolumn{6}{|l|}{ Audit Tool Premises-GMP } \\
\hline Reception-Products inspection & $54,5(12)$ & $45,5(10)$ & $18,2(4)$ & $81,8(18)$ & 0.021 \\
\hline Reception-Conditions of the products & $4,5(1)$ & $95,5(21)$ & $4,5(1)$ & $95,5(21)$ & 0.500 \\
\hline Room Temperature Storage-Separation of food and non-food products & $36,4(8)$ & $63,6(14)$ & $13,6(3)$ & $86,4(19)$ & 0.180 \\
\hline Room Temperature Storage-Organized Stock (FIFO / FEFO) & $31,8(7)$ & $68,2(15)$ & $18,2(4)$ & $81,8(18)$ & 0.453 \\
\hline Room Temperature Storage-Labeling / Products identification & $0,0(0)$ & $100,0(22)$ & $4,5(1)$ & $95,5(21)$ & -- \\
\hline Room Temperature Storage-Non-according products and materials & $86,4(19)$ & $13,6(3)$ & $36,4(8)$ & $63,6(14)$ & 0.003 \\
\hline Room Temperature Storage - Earth products & $22,7(5)$ & $77,3(17)$ & $13,6(3)$ & $86,4(19)$ & 0.688 \\
\hline Cold Storage-Defrosting conditions & $9,1(2)$ & $90,9(20)$ & $4,5(1)$ & $95,5(21)$ & 0.500 \\
\hline Cold Storage-Organized Stock (FIFO / FEFO) & $13,6(3)$ & $86,4(19)$ & $9,1(2)$ & $90,9(20)$ & 0.500 \\
\hline Cold Storage-Freezing Procedure & $45,5(10)$ & $54,5(12)$ & $27,3(6)$ & $72,7(16)$ & 0.344 \\
\hline Cold Storage-Labeling / Products identification & $90,9(20)$ & $9,1(2)$ & $45,5(10)$ & $54,5(12)$ & 0.006 \\
\hline Cold Storage-Packaging & $68,2(15)$ & $31,8(7)$ & $40,9(9)$ & $59,1(13)$ & 0.146 \\
\hline Cold Storage-Non-according products and materials & $13,6(3)$ & 86,4 (19) & $18,2(4)$ & $81,8(18)$ & 0.500 \\
\hline Cold Storage-Temperatures & $13,6(3)$ & $86,4(19)$ & $9,1(2)$ & $90,9(20)$ & 0.500 \\
\hline Cold Storage-Refreezing conditions & $4,5(1)$ & $95,5(21)$ & $0,0(0)$ & $100,0(22)$ & -- \\
\hline Preparation-Preparation conditions & $9,1(2)$ & $90,9(20)$ & $4,5(1)$ & $95,5(21)$ & 0.500 \\
\hline Preparation-Correct handling of prepared foods & $9,1(2)$ & $90,9(20)$ & $9,1(2)$ & $90,9(20)$ & 0.500 \\
\hline Preparation-Proper disinfection of food to consume in raw & $22,7(5)$ & $77,3(17)$ & $18,2(4)$ & $81,8(18)$ & 0.500 \\
\hline Cooking-Handling cooked food & $0,0 \%(0)$ & $100,0(22)$ & $0,0(0)$ & $100,0(22)$ & -- \\
\hline Cooking-Absence of food at room temperature & $36,4(8)$ & $63,6(14)$ & $18,2(4)$ & $81,8(18)$ & 0.388 \\
\hline Cooking-Frying oils conditions & $0,0(0)$ & $100,0(22)$ & $0,0(0)$ & $100,0(22)$ & -- \\
\hline Cooking-Presence of leftovers and scraps & $31,8(7)$ & $68,2(15)$ & $13,6(3)$ & $86,4(19)$ & 0.289 \\
\hline Cooking-Sampling & $0,0(0)$ & $100,0(22)$ & $0,0(0)$ & $100,0(22)$ & -- \\
\hline Cooking-Eve cooking & $13,6(3)$ & $86,4(19)$ & $9,1(2)$ & $90,9(20)$ & 0.500 \\
\hline Serving-Food exposure conditions & $13,6(3)$ & $86,4(19)$ & $9,1(2)$ & $90,9(20)$ & 0.500 \\
\hline Serving-Exposure temperature & $9,1(2)$ & $90,9(20)$ & $4,5(1)$ & $95,5(21)$ & 0.500 \\
\hline Serving-Plating procedure & $0,0(0)$ & $100,0(22)$ & $0,0(0)$ & $100,0(22)$ & -- \\
\hline Serving-Existence of non-compliant products & $0,0(0)$ & $100,0(22)$ & $0,0(0)$ & $100,0(22)$ & -- \\
\hline
\end{tabular}


integrity of containers and expiry date and no records kept for control and tracking, like in Veiros, et al. [7] but with the FoodSimplex there was a major evolution towards food safety.

In storage at room temperature, changes in product identification and labeling for compliance were also statistically significant $(\mathrm{p}=0.003)$. Initially, only $13.6 \%$ were satisfied with the item and after the intervention, 14 companies came to fulfill the requirements (63.6\%). Of the 19 non-compliant restaurants $(86.4 \%)$, only 8 maintained noncompliance in the fourth phase (36.4\%).

The same happened for the labeling and identification of products in cold storage under conditions ( $\mathrm{p}$-value $=0.006$ ), with $90.9 \%$ noncompliances in the first evaluation and at the end of the 4 th audit there was a decrease to $54.5 \%$ restaurants.

In Veiros, et al. [7] the cold storage presented nonconformities as well, also with visible labeling. The remaining items evaluated through the audit tool evolved to conformance between the first and last audit. In Garayoa, et al. [3], deficiencies were detected, namely: in the treatment of leftovers, which was not verified in this study, with compliance being complied with in $100 \%$; in thawing of products, which also did not occur in this work since compliance in the first phase was $90.9 \%$ with an increase in compliance to $95.5 \%(n=21)$; and disinfection of vegetables (95\%), which in this investigation started with $77.3 \%$ compliance and ended with an increase of $81.8 \%(n=18)$.

\section{Restaurants - hygiene and GMP}

The results of the $1^{\text {st }}$ audit highlighted significant gaps in knowledge, attitudes and practices of safe food handling in restaurants. The areas of high concern were: the poor hygiene of cold storage, cooking, locker rooms, handwashing sinks, $1^{\text {st }}$ aid kit, dishwashers and waste treatment. In GMP were receiving inspection, labeling in room temperature and cold storage, freezing procedures and cold packaging of food. All these items presented nonconformities in more than $50 \%$ of the restaurants.

After the application of FoodSimplex, the restaurants $(n=22)$ showed an evolution in compliance both in hygiene and GMP.

In Figure 1, we found that for Hygiene, there was a statistically significant change for compliance for all companies $(\mathrm{p}=0.01)$. Reiterating that the FoodSimplex application has positive results for all hygiene items.

In Figure 2, there was also a statistically significant change to the compliance for all GMP companies $(p=0.005)$, with the Food Simplex method also suitable for this group of items.

The reasons some restaurants still find struggle to comply the food safety requirements is the reduced number of foodservice workers and the time constraints in the restaurants.

FoodSimplex was designed to address all the technical barriers that small and medium-sized catering companies faced in preimplementation, implementation and after the implementation of the HACCP system [14].

One of the differentiating factors in the methodology is that it communicates the audit's results and corrective measures through training to all food handlers as well as to the restaurants FBOs. Which proved to be a methodological step of high importance, also according to the study of Powel, et al. [2] "Audit reports are only useful if the purchaser who requires them to review the results, understands the risks addressed by the standards and makes risk-reduction decisions based on the results".

\section{RESTAURANTS HYGIENE EVOLUTION}

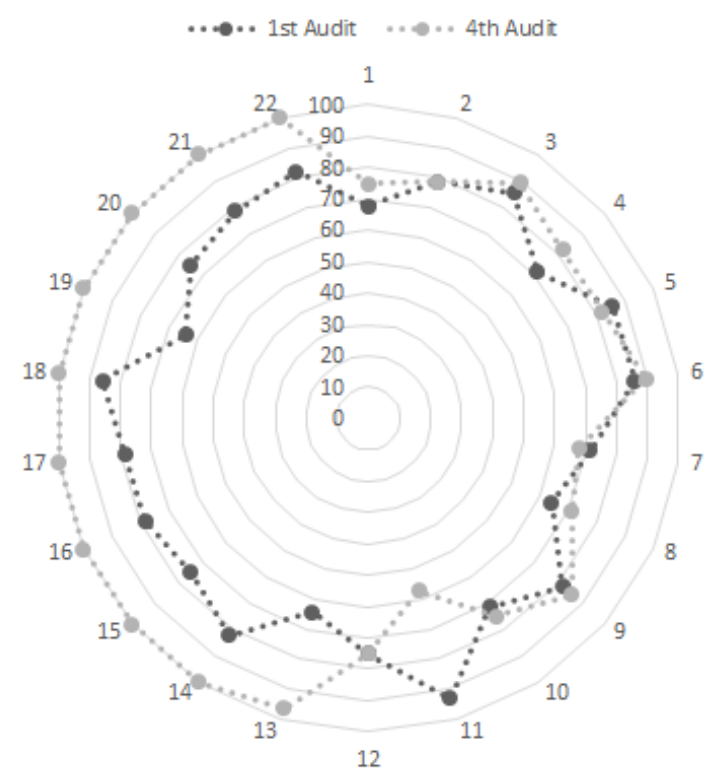

Figure 1. Restaurants hygiene evolution

\section{RESTAURANTS GMP EVOLUTION}

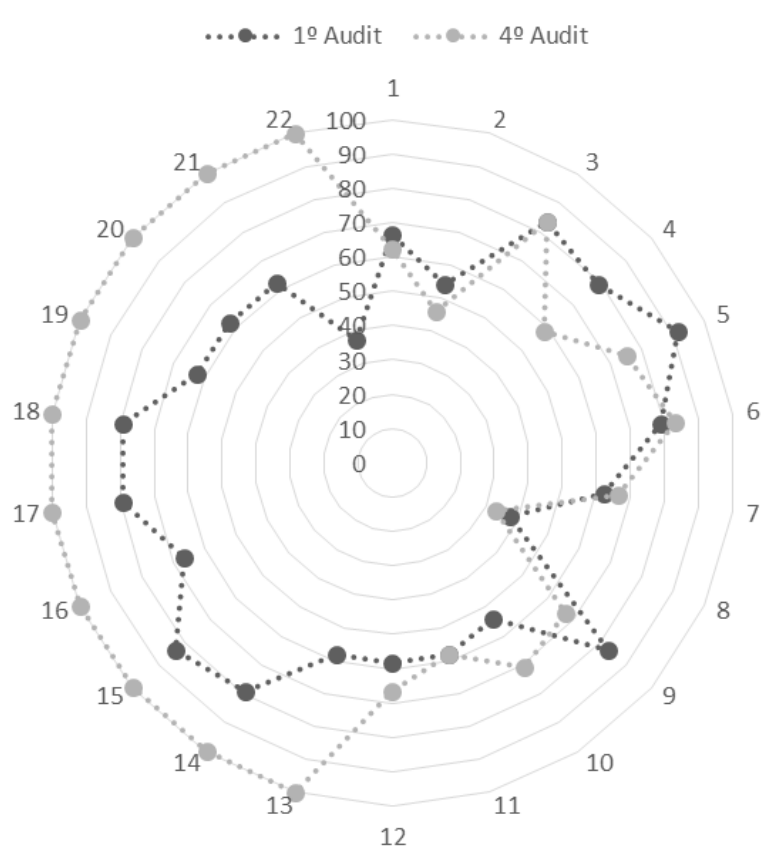

Figure 2. Restaurants GMP evolution

FoodSimplex also defines time for handling the audit nonconformities detected and sets the time for their correction, knowing the team will be re-evaluated in the next audit. The study by LäikköRoto, et al. [22], also defends the significance of time limit for corrective measures on the efficacy of the controls.

One of the limitations that we can point out to the study is that the audit tool (or audit checklist) is not valid since there is no scientific basis for certification/validation in audits. There is high variability in the quality and reliability of audits and many different types of audit 
tools that vary in length, complexity, and style [2]. In the study, the researchers try to develop a checklist according to the stages of the meals production, as well as assess the main 3 groups (hygiene, GMP, and HACCP documentation), with a quantitative evaluation and allowing a periodic application to monitoring the restaurants.

\section{Conclusions}

The primary causes of food poisoning in restaurants are cross contamination between raw and cooked foods; insufficient heating; keeping food at room temperature for extended periods of time; contamination by infected food handlers and contamination by inadequately cleaned equipment [23].

The FoodSimplex method allowed us the possibility of assessing food handlers and of exploring how the action plan was useful in changing behaviors towards food safety.

These gaps were resolved with risk-based training of food handlers in the facilities, using appropriate training aids to encourage understanding and assurance in the application of food safety principles in their day-to-day operations.

In this work, the FoodSimplex was linked to a change of habits to obtain a sustained improvement in food safety. It is important to refer that, after continuous and systematic training, with frequent audits, the hygiene and the GMP in these restaurants has improved. As so, this methodology proved to be efficient in public health matters, namely in the production of safer meals in SME restaurants.

\section{References}

1. Regulation (EC) No. 1169/2011. The European Parliament and the Council of 25th October 2011

2. Powell DA, Erdozain S, Dodd C, Costa R, Morley K, et al. (2013) Audits and inspections are never enough: A critique to anhance food safety. Food Control 30: 686-691.

3. Garayoa R, Vitas AI, Díez-Leturia M, García-Jalón I (2011) Food Safety and the contract catering companies: Food handlers, facilities and HACCP evaluation. Food Control 22: 2006-2012.

4. Medeiros CO, Cavalli SB, Proença RPC (2012) Human resources administration processes in commercial restaurants and Food Safety: the action of administrators. Int J Hosp Manag 31: 661-674.

5. Baptista P, Linhares M (2005) Hygiene and Food Safety in Catering: Volume I Initiation. Forvisão. Guimarães, Portugal.
6. Bank of Portugal (2011) Sector Analysis of accommodation, catering and alike. Studies of central balances. Lisbon, Portugal.

7. Veiros MB, Proença RPC, Santos MCT, Rocha A, Kent-Smith L (2007) Proposta de check-list hígio-sanitária para unidades de restauração. Alimentação Humana 13: 51-61.

8. APHORT (2008) Code of Good Practices in Food Safety. Portuguese Association of Hotels, Restaurants and Tourism, Portugal.

9. Codex Alimentarius (2003) Recommended International code of practice - General Principles of Food Hygiene. CAC/RCP 1-1969 Rev 4.

10. Sun Y, Ockerman HW (2005) A review of the needs and current applications of hazards analysis and critical control point (HACCP) system. Food Control 16: 325-332.

11. Regulation (EC) No. 852/2004. The European Parliament and the Council of 29th April 2004.

12. Forsythe SJ, Hayes PR (1998) Food Poisoning and other foodborne hazards. In Forsythe SJ, Hayes, PR (eds). Food hygiene, microbiology and HACCP. Third edition. Gaithersburg, MD: Aspen Publication, pp: 21-85.

13. Charalambous M, Fryer PJ, Panayides S, Smith M (2015) Implementation of Food Safety Management Systems in small food businesses in Cyprus. Food Control 57: $70-75$.

14. Panisello PJ, Quantick PC (2001) Technical barriers to Hazard Analysis Critical Control Point (HACCP). Food Control 12: 165-173.

15. Haukijärvi V, Lundén J (2017) Does waiving preventive food control inspections in Filand weaken the prerequisites for safe food handling in restaurants? Food Control 71: 187-192.

16. ANZFA (2001) Food Safety: an audit system. Australian New Zealand Food Authority. www.info@anzfa.gov.eu

17. Nunes JL, Curto H, Varela M (2016) Gestão de Tesouraria: O impacto da atual crise económica e financeira nas PME portuguesas. Business and Economics School, Lisbon.

18. http://hdl.handle.net/10400.26/15250

19. PORDATA (2017) Pequenas e médias empresas: total e por sector de atividade económica. Base de Dados Portugal Contemporâneo. https://www.pordata.pt

20. Sarter G, Sarter S (2012) Promoting a culture of food safety to improve hygiene in small restaurants in Madagascar. Food Control 25: 165-171.

21. Veiros MB, Proença RPC, Santos MCT, Kent-Smith L, Rocha A (2009) Food safety practices in a Portuguese canteen. Food Control 20: 936-941.

22. Santos MC (2005) Segurança alimentar Porto/EURO 2004. Universidade Técnica de Lisboa, Lisboa.

23. Läikkö-Roto T, Mäkelä S, Lundén J, Heikkilä J, Nevas M (2015) Consistency in inspection processes of food control officials and efficacy of official controls in restaurants in Finland. Food Control 57: 341-350

24. Ko W (2015) Food suppliers' perceptions and practical implementation of food safety regulations in Taiwan. J Food Drug Anal 23: 778-787.

Copyright: (C2017 Baltazar A. This is an open-access article distributed under the terms of the Creative Commons Attribution License, which permits unrestricted use, distribution, and reproduction in any medium, provided the original author and source are credited. 